\title{
Impact and relationship of childhood experiences and substance abuse in a population of Baghdad City, Iraq
}

\author{
Riyadh K Lafta ${ }^{1}$, Ameel F Al-Shawi ${ }^{2}$, Ahmed Samir Al-Nuaimi ${ }^{3}$, Saleh Al Hasnawi ${ }^{4}$ \\ ${ }^{1}$ Professor, College of Medicine/ Mustansiriya University. Iraq (Affiliate Professor, University of Washington, USA). ${ }^{2}$ Lecturer, \\ Department of Community Medicine, College of Medicine/Mustansiriya University. ${ }^{3}$ Assistant Professor, Department of \\ Community Medicine, College of Medicine/Baghdad University. ${ }^{4}$ Professor, Department of Psychiatry, College of Medicine, \\ Kerbala University, Iraq (Iraqi Congress member, Committee of Health; Ex-Minister of Health, Iraq)
}

\begin{abstract}
Adverse childhood experiences (ACEs) (e.g. abuse, neglect, violence between parents or caregivers, and community violence) are associated with higher rates of depression, tobacco use, alcoholism, illicit drug use and attempted suicide among adult population. The objective of this study is to identify the relationship of childhood experiences to substance use during adulthood in a sample from Baghdad city. A multistage sampling technique was used to choose respondents from primary health care centers and universities. Childhood experiences were measured by applying a modified standardized Adverse Childhood Experiences International Questionnaire (ACE-IQ) to inquire about the negative childhood experiences (household dysfunction and abuse, and exposure to community and collective violence) and positive childhood experiences presented by bonding to family. A total of 1040 subjects were surveyed and 1000 responded, making a response rate of $96.2 \%$. The mean score of household dysfunction and abuse is significantly higher among those with a positive history of taking sedative drugs (24.1) compared to those with a negative history (12.3). The association between sedative drugs use and the score of household dysfunction and abuse is rated as a strong association. A strong association was found between history of alcohol drinking and a higher score of household dysfunction and abuse. The mean score of bonding to family (76.2) is significantly higher among those with a negative history of sedative drug use compared to the mean score of bonding to family of subjects with a positive history of sedative drug use (65.3). It can be concluded from this study that sedative drug use is strongly associated with household dysfunction and abuse, the same is for alcohol drinking. Special national programs, including prevention and intervention strategies, are needed to build resilience among people targeting early adverse childhood experiences and their consequences.
\end{abstract}

Keywords: Childhood experiences, Household dysfunction, Violence, Bonding to family, Substance use.

\section{Introduction}

Adverse Childhood Experiences (ACEs) refer to some of the most intensive and frequently occurring sources of stress that children may suffer early in life. Such experiences include multiple types of abuse; neglect; violence between parents or caregivers; community and collective violence. ${ }^{1}$

The ACEs literatures show that exposure to multiple risk factors during childhood is associated with higher rates of depression, tobacco use, alcoholism, illicit drug use, and attempted suicide. ${ }^{2-4}$ ACEs are associated with significant functional impairment and life loss in adolescence and adulthood. ${ }^{5}$ The family is one of the most critical risks and resilience variables for substance abuse in adolescence and emerging adulthood. ${ }^{6}$ The most consistently reported variables that facilitate positive adaptation under the conditions of risk are connections with competent caring adults, self-regulation skills, and positive self-views. ${ }^{7}$

Iraq is an example of the challenging mental health needs in low-income, conflict-affected countries as it has been consistently exposed to large-scale traumatic events such

\section{Practice Points}

- ACEs refer to some of the most intensive and frequently occurring sources of stress that children may suffer early in life.

- Iraq has been consistently exposed to largescale traumatic events which negatively impacts the psychosocial status of the people, specially children.

- This study showed a strong association between sedative drugs use and household dysfunction and abuse. Family bonding during childhood are found to be the most predictive resilient variable that protects adults against substance use, mental disorders, and suicidal attempts.

- High self-esteem has an inverse relationship with depression and anxiety symptoms, suicidal attempts and substance abuse.

- Special national programs, including prevention and intervention strategies, are needed to build resilience among people targeting early adverse childhood experiences and their consequences.

Correspondence: Professor Saleh Al Hasnawi, Department of Psychiatry, College of Medicine, Kerbala University, Iraq. Email: shasnawi@yahoo.com. 
as successive wars (since 1980 to present), economic sanctions, sustainable organized violence, and terrorism. This unsafe situation negatively impacts on the psychosocial status of the whole Iraqi community, ${ }^{8-11}$ especially children and youth who have been so greatly affected by this condition through facing disease, psychological trauma and death. ${ }^{11-14}$

There have been many reports about the violence in Iraq during the years of intense conflict; however, disabilities and mental health trauma from these years have not been widely documented. ${ }^{15}$ The extent of psychological trauma in the population has led the Ministry of Health to include mental health in the basic health services package. ${ }^{16}$ Although some pilot community psychosocial services have been implemented, how these services will be linked to the basic health services package if shown to be successful is unclear. ${ }^{11}$

\section{Materials and Methods}

We designed the cross sectional study as a retrospective cohort by dealing with the exposure as the independent variable. The study was conducted in Baghdad city during the period from January 2013 through January 2014.

Study sites:

The sample was collected mainly from two sources:

- Primary health care centers (PHCCs): a multistage random sampling technique was used. Baghdad is divided into 16 health sectors, out of these; five sectors were chosen by a simple random technique. The total number of PHCCs in these five sectors was 60; two-three PHCCs were chosen from each sector according to the density of its distribution, so, 13 PHCCs from the two main sides of Baghdad city were collected that represent central and peripheral sectors. Each PHCC was visited for 2-3 weeks to collect data from daily attendants through a systematic random sampling technique by including every fourth one.

- Universities: A multistage random sampling technique was adopted by selecting three universities out of the five that are present in Baghdad through a simple random sampling technique, then three colleges -from each university- were selected by a simple random sampling technique, and one stage from each college again by a simple random technique; all students of that stage, who were available at the time of data collection, were included in the sample.

\section{Sample size/ sample population:}

The target population was males and females aged between 18-59 years in order to widen the spectrum and to increase the number of end points. Individuals age 60 years and more were not included to minimize the effect of recall bias.

As there is no previous study in Iraq about the prevalence of substance use that is related to the effect of ACEs, so we could not calculate the sample size based on prevalence. Considering the time limit; we planned to take a sample size of not less than 1000 subject (both genders) to minimize the role of chance, on the other hand, it was not feasible to collect a larger sample size considering the insecurity situation. Subjects who were not raised in Baghdad were not included.

Data collection was done by two well-trained community physicians through distributing a selfreported questionnaire to the interviewees after giving them a short explanation about the questions, taking their verbal consent to participate and assuring them that all the information will be kept strictly confidential and will not be used for anything other than research purposes.

\section{Instruments:}

The questionnaire consisted of the following items:

a. Socio-demographic information: Age (18-59 years), current education level, history of smoking habits and alcohol drinking whether previously or currently.

b. Adverse childhood experiences (when the age was 15 years or less) including:

$\diamond$ Household dysfunction and abuse.

$\diamond$ Exposure to community and collective violence.

Adverse childhood experiences were measured by applying a modified standardized Adverse Childhood Experiences International Questionnaire (ACE-IQ) form that was developed by WHO, ${ }^{1}$ and includes:

- Categories of household dysfunction and abuse: psychological abuse, physical abuse, household dysfunction including violence against mother or other household members, living with household members who are (substance abusers, mentally ill or suicidal), ever imprisoned, and parent's loss during childhood.

- Witnessing community violence that includes: seeing or hearing someone being beaten, stabbed or shot in real life.

- Exposure to collective violence including wars, terrorism, ethnic conflicts, repression, disappearance and torture; this was measured via the following questions: forced to go and live in another place, if a family member or a friend was kidnapped, killed or beaten up by soldiers, police, militia, or gangs.

c. Positive childhood experiences: were indicated by bonding to family and parental monitoring (when the age was 15 years and less):

Bonding to family was measured by a modified five items derived from an instrument ${ }^{17,18}$ and questions about relationship with parents that are presented in ACE-IQ, ${ }^{1}$ the subjects indicated how much they would like to be the kind of people their parents were, how much their parents made them feel trusted, how much they depended on their parents for advice and guidance, how much the parents understood their problems and worries.

Responses for questions of bonding to family range from "strongly disagree" to "strongly agree" on a 
four point scale. Three items for parental monitoring were put as indicators: time spent talking about school and other activities of the day, time spent playing with the subjects and knowing (who) their friends are. Possible responses for parental monitoring items ranged from "almost never" to "often. ${ }^{17}$

\section{d. History of substance use includes:}

- History of smoking habits: previous or current smoking, age starting smoking

- History of alcohol drinking: previous or current and age of starting drinking.

- Sedative drug use including: sedative, psychotic, narcotic and hypnotic drugs.

\section{Data Analysis:}

Descriptive and analytic statistics were performed using the Statistical Package for Social Science (SPSSversion 21) program.

- The score for bonding to family and for parental monitoring was calculated as a single score.

- Variables of alcohol drinking and smoking includes current and previous history.

- Standardization scores of household dysfunctionabuse and family bonding score were calculated to each participant according to the following equation:

Standardization score $(/ 100)=\operatorname{sum}(\mathrm{Q} 1$ to $\mathrm{Q} n) \mathrm{X}$ $100 /$ ) count valid * upper limit of scoring of the questions in the scale).

$\diamond \quad \operatorname{Sum}(\mathrm{Q} 1$ to $\mathrm{Q} n)=$ summation of questions answers for that scale.

$\diamond$ Count valid $=$ number of answered questions of that scale.

$\checkmark$ Standardization of the scores was to bypass the effect of missed questions, and to give unique way in the analysis (all scores started from zero to 100).

- Cronbach's Alpha reliability was measured for the scales, the results were:

$\diamond$ Cronbach's Alpha reliability of bonding to family scale was: 0.86 (strong). Cohen's (d) was used to estimate the effect size for independent samples t-tests. ${ }^{19}$ The interpretation of Cohen's (d) is as follows: ${ }^{11}$

- Cohen's (d) up to 0.3 is considered as small effect size.

- Cohen's (d) more than 0.3 to 0.7 is considered a medium effect size.

- Cohen's (d) more than 0.7 is considered as large effect size.

\section{Results}

Description of the study sample

A total of 1040 subjects were surveyed and 1000 responded making a response rate of $96.2 \%$. The respondents' age ranged from 18 to 59 years with a mean of $32.08 \pm 11.169$, females constituted a higher proportion $(58.3 \%)$. Only $18.5 \%$ of the participants reported smoking, $4.2 \%$ had history of alcohol drinking and $4.8 \%$ had a history of using sedative drugs. The other socio-demographic characteristics of the sample are summarized in Table 1.

Exposure to adverse childhood experiences

Exposure to household dysfunction and abuse: Table 2 shows that father's death (when the subject's age was less than 15 years) was seen in $10.4 \%$ of the participants, while mother's death was seen in $2.1 \%$ of them. Parents' separation was registered in 3\% of the subjects. Seeing or hearing a parent or household member in home being yelled at, screamed at, sworn at, insulted or humiliated was reported in $46.9 \%$. Seeing or hearing a parent or household member at home being slapped, kicked, punched or beaten up was seen in $33.1 \%$, seeing or hearing a parent or household member in home being hit or cut with an object, such as a stick, bottle, club, knife or whip was reported in $17.5 \%$. A parent, guardian or other household member yelled, screamed or swear at the respondents, insulted or humiliated them, was registered in $38.7 \%$. A parent, guardian or other household member spanked, slapped, kicked or punched the subject, was seen in $33.5 \%$. (All items in Table 2 represent responses of sometimes and frequently).

Exposure to Community Violence (age below 15 years): As shown in Table 3, the most common trauma event of community violence which was reported by the participants was seeing or hearing someone being beaten up in real life $(48.3 \%)$. Seeing

Table 1: Socio-demographic characteristics of the respondents

\begin{tabular}{|l|c|}
\hline \multicolumn{1}{|c|}{ Variables } & Respondents (\%) (n-1000) \\
\hline Gender & $583(58.3 \%)$ \\
\hline Female & $417(41.7 \%)$ \\
\hline Male & $498(49.9 \%)$ \\
\hline Age group (years) & $227(22.7 \%)$ \\
\hline$<30$ & $177(17.7 \%)$ \\
\hline $30-39$ & $96(9.6 \%)$ \\
\hline $40-49$ & $135(13.5 \%)$ \\
\hline $50-59$ & $127(12.7 \%)$ \\
\hline Highest level of education completed \\
\hline Primary school & $122(12.2 \%)$ \\
\hline Intermediate & $603(60.4 \%)$ \\
\hline Secondary & $12(1.2 \%)$ \\
\hline University/Diploma & $814(81.5 \%)$ \\
\hline Post graduate & $185(18.5 \%)$ \\
\hline Cigarettes smoking & \\
\hline Non smoker & $949(95.8 \%)$ \\
\hline Ever smoked & $42(4.2 \%)$ \\
\hline Alcohol drinking habit & $837(95.2 \%)$ \\
\hline Never drank & $42(4.8 \%)$ \\
\hline Ever drank & \\
\hline Use of sedative drugs & No \\
\hline Yes & \\
\hline \multicolumn{2}{|c|}{} \\
\hline
\end{tabular}

*The difference in the totals is according to the response of the participants to each question 
Table 2: Frequency distribution of household dysfunction and abuse items (age below 15 yrs)

\begin{tabular}{|l|c|}
\hline \multicolumn{1}{|c|}{ Household dysfunction and abuse items } & $\begin{array}{c}\text { Respondents (\%) } \\
(\mathrm{n}-1000)\end{array}$ \\
\hline Father died when the subject was $<15$ years old & $104(10.4 \%)$ \\
\hline Mother died when the subject was $<15$ years old & $21(2.1 \%)$ \\
\hline Parents separated when the subject was $<15$ years of age & $30(3.0 \%)$ \\
\hline $\begin{array}{l}\text { Live with a household member who was a problem drinker, alcoholic, or misused street or } \\
\text { prescription drugs }\end{array}$ & $133(13.3 \%)$ \\
\hline Lived with a household member who was depressed, mentally ill or suicidal & $83(8.3 \%)$ \\
\hline Lived with a household member who was ever sent to jail or prison & $105(10.5 \%)$ \\
\hline $\begin{array}{l}\text { Saw or heard a parent or household member in home being yelled at, screamed at, sworn at, } \\
\text { insulted or humiliated }\end{array}$ & $469(46.9 \%)$ \\
\hline $\begin{array}{l}\text { Saw or heard a parent or household member in home being slapped, kicked, punched or } \\
\text { beaten up }\end{array}$ & $331(33.1 \%)$ \\
\hline $\begin{array}{l}\text { Saw or heard a parent or household member in home being hit or cut with an object (stick, } \\
\text { bottle, club, knife, whip etc.) }\end{array}$ & $175(17.5 \%)$ \\
\hline $\begin{array}{l}\text { If a parent, guardian or other household member threaten to, or actually, abandon you or } \\
\text { throw you out of the house }\end{array}$ & $137(13.7 \%)$ \\
\hline $\begin{array}{l}\text { If a parent, guardian or other household member yelled, screamed, at you, insulted or } \\
\text { humiliated you }\end{array}$ & $387(38.7 \%)$ \\
\hline If a parent or other household member did spank, slap, kick, punch or beat you up & $162(16.2 \%)$ \\
\hline $\begin{array}{l}\text { If a parent, guardian or other household member hit or cut you with an object, such (stick, } \\
\text { bottle, club, knife, whip etc.) }\end{array}$ & $335(33.5 \%)$ \\
\hline If bad treatment resulted in injury & $33(3.3 \%)$ \\
\hline
\end{tabular}

or hearing someone being threatened with a knife or gun in real life was reported in $18.1 \%$. A family member or friend kidnapped or beaten up by soldiers, police, militia, or gangs seen in $14.8 \%$. A family member or friend killed by soldiers, police, militia, or gangs reported in $17.2 \%$. (All items in Table 3 represent responses of once and frequently).

Bonding to family (age below 15 years): Table 4 shows that $74.8 \%$ of the subjects like to be the kind of people their parents were, the parents made them feel trusted in $83.4 \%$, and $77.5 \%$ of the participants have parents who understood their problems and needs, parents spent time talking with the subjects about activities of the day and playing with them during childhood and adolescence were reported in $69.2 \%$. (All items in Table 4 represent responses of agree and strongly agree).

Relationship of household dysfunction and abuse with substance use: As shown in Table 5; the mean score of household dysfunction and abuse is significantly higher among those with positive history of taking sedative drugs (24.1) compared to those with a negative history (12.3). The association between sedative drugs use and score of household dysfunction and abuse is rated as strong association (Cohen's $d>0.8$ ), a strong association

Table 3: Frequency distribution of exposure to community violence items (age below 15 years)

\begin{tabular}{|l|c|}
\hline \multicolumn{1}{|c|}{ Community Violence (age below 15 years) } & $\begin{array}{c}\text { Respondents (\%) } \\
(\mathrm{n}-1000)\end{array}$ \\
\hline Exposed to bullying & $176(17.6 \%)$ \\
\hline Saw or heard someone being beaten up in real life & $483(48.3 \%)$ \\
\hline Saw or heard someone being threatened with a knife or gun in real life & $181(18.1 \%)$ \\
\hline Forced to go and live in another place & $107(10.7 \%)$ \\
\hline Beaten up by soldiers, police, militia, or gangs & $27(2.7 \%)$ \\
\hline A family member or friend kidnapped or beaten up by soldiers, police, militia, or gangs & $148(14.8 \%)$ \\
\hline A family member or friend killed by soldiers, police, militia, or gangs & $172(17.2 \%)$ \\
\hline
\end{tabular}

Table 4: Frequency distribution of the items of bonding to family (age below 15 years)

\begin{tabular}{|l|c|}
\multicolumn{1}{|c|}{ Bonding to family } & $\begin{array}{c}\text { Respondents (\%) } \\
(\mathrm{n}-1000)\end{array}$ \\
\hline Like to be the kind of people as his/her parents were & $748(74.8 \%)$ \\
\hline Parents made them feel trusted & $834(83.4 \%)$ \\
\hline Parents understood their problems and needs & $775(77.5 \%)$ \\
\hline They depended on their parents for advice and guidance & $835(83.5 \%)$ \\
\hline Parents encouraged me for going to school & $917(91.7 \%)$ \\
\hline Parents spent time talking with the participants about school & $805(80.5 \%)$ \\
\hline $\begin{array}{l}\text { Parents spent time talking with the participants about activities of the day and spent time } \\
\text { for playing and travels }\end{array}$ & $692(69.2 \%)$ \\
\hline Parents knew the friends of their sons/daughters (participants) & $906(90.6 \%)$ \\
\hline
\end{tabular}

South East Asia Journal of Public Health 2015;5(1):25-32 
Table 5: Scores of household dysfunction and abuse (/100) in relation to substances use

\begin{tabular}{|c|c|c|c|c|c|c|}
\hline \multirow[t]{2}{*}{ Variables } & \multicolumn{5}{|c|}{ Scores $(/ \mathbf{1 0 0})$} & \multirow{2}{*}{$\begin{array}{c}\text { Cohen's } \\
\text { d } \\
\end{array}$} \\
\hline & Mean & SD & SE & $\mathbf{N}$ & $\mathbf{P}$ & \\
\hline \multicolumn{7}{|l|}{ Smoking cigarettes } \\
\hline Non smoker & 11.9 & 13.5 & 0.47 & 814 & 0.003 & 0.25 \\
\hline Ever smoked & 15.3 & 14.1 & 1.04 & 185 & & \\
\hline \multicolumn{7}{|l|}{ Alcohol drinking habit } \\
\hline Never drank & 12.1 & 13.3 & 0.43 & 949 & $<0.001$ & 0.71 \\
\hline Ever drank & 21.6 & 15.3 & 2.36 & 42 & & \\
\hline \multicolumn{7}{|l|}{ Being on sedative drugs } \\
\hline No & 12.3 & 13.3 & 0.46 & 837 & $<0.001$ & 0.87 \\
\hline Yes & 24.1 & 18 & 2.77 & 42 & & \\
\hline
\end{tabular}

was found between history of alcohol drinking and higher scores of household dysfunction and abuse (Cohen's d $>0.7$ ).

Smoking habit is significantly associated with a higher mean score of household dysfunction and abuse (15.3) compared to nonsmoking (11.9), however, the association between smoking habit and score of household dysfunction and abuse is rated as a small effect size (Cohen's $\mathrm{d}=0.25)$.

Relationship of community violence exposure with substances use: Table 6 reveals that the mean score of community violence exposure is significantly higher among males (25.1) compared to females (14.6), there is a moderate association between gender and score of community violence exposure (Cohen's $d=0.51$ which is considered as moderate effect size). Positive history of sedative drugs use shows moderate association with mean score of community violence exposure (Cohen's $\mathrm{d}=0.4$ ). There is a statistically insignificant association between alcohol drinking and score of community violence exposure $(p>0.05)$.

Relationship of family bonding score and substance use: Table 7 shows that the mean score of bonding to family is significantly higher among those with a negative history of sedative drug use (76.2) compared to the mean score of bonding to family of subjects with a positive history of sedative drug use (65.3). Positive history of using sedative drugs shows a moderate inverse association with the mean score of bonding to family (Cohen's $d=-0.51$ which is considered as a medium effect size).

\section{Discussion}

A house to house survey would be a good choice in such studies but this is extremely insecure in a conflict country like Iraq under the current circumstances. The sample looks relatively skewed towards the "high education" this is due to that about half the sample was taken from university students and the other half from

Table 6: Scores of exposure to community violence (/100) in relation to substances use

\begin{tabular}{|c|c|c|c|c|c|c|}
\hline \multirow[t]{2}{*}{ Variables } & \multicolumn{5}{|c|}{ Scores (/100) } & \multirow[t]{2}{*}{ Cohen's d } \\
\hline & Mean & SD & SE & $\mathbf{N}$ & $\mathbf{P}$ & \\
\hline \multicolumn{7}{|l|}{ Smoking cigarettes } \\
\hline Non smoker & 18.2 & 20.7 & 0.73 & 796 & 0.016 & 0.21 \\
\hline Ever smoked & 22.6 & 22.6 & 1.67 & 183 & & \\
\hline \multicolumn{7}{|l|}{ Alcohol drinking habit } \\
\hline Never drank & 18.8 & 21.1 & 0.69 & 930 & 0.17 & 0.22 \\
\hline Ever drank & 23.5 & 21 & 3.23 & 42 & & \\
\hline \multicolumn{7}{|l|}{ Being on sedative drugs } \\
\hline No & 18.1 & 20.8 & 0.72 & 820 & 0.017 & 0.4 \\
\hline Yes & 26.5 & 21.5 & 3.32 & 42 & & \\
\hline
\end{tabular}

Table 7: Scores of bonding to family (/100) in relation to substances use

\begin{tabular}{|c|c|c|c|c|c|c|}
\hline \multirow[t]{2}{*}{ Variables } & \multicolumn{5}{|c|}{ Score of bonding to family $(/ \mathbf{1 0 0})$} & \multirow[t]{2}{*}{ Cohen's d } \\
\hline & Mean & SD & SE & $\mathbf{N}$ & $\mathbf{P}$ & \\
\hline \multicolumn{7}{|l|}{ Smoking cigarettes } \\
\hline Non smoker & 74.9 & 21.4 & 0.75 & 813 & 0.9 & 0.01 \\
\hline Ever smoked & 75.1 & 20.4 & 1.5 & 185 & & \\
\hline \multicolumn{7}{|l|}{ Alcohol drinking habit } \\
\hline Never drank & 75.2 & 21.1 & 0.69 & 948 & 0.16 & -0.23 \\
\hline Ever drank & 70.3 & 22 & 3.4 & 42 & & \\
\hline \multicolumn{7}{|l|}{ Being on sedative drugs } \\
\hline No & 76.2 & 21 & 0.73 & 837 & 0.01 & -0.51 \\
\hline Yes & 65.3 & 25.7 & 3.97 & 42 & & \\
\hline
\end{tabular}

South East Asia Journal of Public Health 2015;5(1):25-32 
the general population (PHC attendants), illiterate people were not included as we noticed (during the pilot study) that they faced some difficulties in understanding and responding to some of the questions. On the other hand; age, as a confounder, could not be completely evaluated, people who are in their fifties (for example) might not have the same childhood experiences as those who are now in their third decade because of differences in norms between generations.

\section{Substance use:}

The frequency of smoking habits in the current study among the participants was $18.5 \%$, the literatures revealed that the prevalence of smoking in Iraq in the last ten years ranged between $5 \%$ and $47 \%$ among males and $1-10 \%$ among females. The reasons behind these wide ranges are mostly related to the methods used in the surveys, for example, household surveys collect information about all occupants from one person in the household. ${ }^{20}$

Frequency of alcohol drinking (4.2\%) and of drug use (4.8\%) among the participants is higher than what was reported in the Iraqi Mental Health Survey (IMHS) $(0.7 \%$ and $0.2 \%)$ respectively, ${ }^{21}$ this might be attributed to the differences in the method, questionnaire and sampling techniques; however, there is a sort of underestimation in the prevalence of substance use among the Iraqi population due to the fact that many respondents deny having this habit as it is considered a sensitive and unaccepted norm in the Iraqi culture, besides, scientific literature which document substance use are scarce and limited. ${ }^{20}$

\section{Household dysfunction-abuse and substance use:}

The results showed that the mean score of household dysfunction and abuse is significantly higher among those with a positive history of sedative drugs use and of alcohol drinking compared to those with a negative history, the association was rated as large effect size indicating that exposure to domestic violence during childhood makes the subject less resilient and increases stress feeling during adulthood leading him to use alcohol or sedative drugs. This finding is consistent with what was reported in literatures as there is a strong graded relationship between ACE categories with alcoholism and drug abuse. ${ }^{22-24}$

The mean score of household dysfunction-abuse was significantly higher among subjects with history of smoking habit, however, the association between smoking and score of household dysfunction-abuse was rated as a small effect size; the positive association indicates that ACEs increase the risk of smoking, and this is consistent with what was reported in the literatures as use of nicotine has been linked to self-medicating efforts to cope with negative emotional, neurobiological, and social effects of ACEs, ${ }^{25}$ on the other hand, the small effect size could be attributed to a point that the smoking behavior among youth is affected by several factors such as effect of peer relationship, general community conditions and society culture, given the fact that there was an underestimation of smoking habits.

\section{Community violence exposure and substance use:}

The mean score of community violence exposure showed a moderate association with positive history of sedative drugs use. This agrees with what was reported in the literatures as that exposure to community violence increases the risk for drug abuse. ${ }^{23}$ There is a statistically insignificant association between smoking habit and alcohol drinking with a score of community violence exposure, this finding might be interpreted by the fact that the exposure to community violence occurred during childhood (age less than 15 years) and the effect of trauma on children depends on many factors such as age, self-esteem, nature of stress, developmental level, personality, religious affiliation, availability of social support, a nurturing family and culture; the culture influences how people react to different cognitive appraisals so that reactions generally correspond and reinforce cultural norms, ${ }^{26}$ many researches stated that intellectual skills and social cognitive abilities functions are protective factors. ${ }^{27,28}$

\section{Family bonding and substance use:}

The mean score of family bonding was significantly higher among those with a negative history of using sedative drugs compared to subjects with a positive history. Mean score of bonding to family showed a moderate inverse relationship with the positive history of using sedative drugs. This finding is consistent with what was reported in the literatures as that bonding to family is considered as a strong predictor of resilience and the resilient subjects have lower tendency for substance use. ${ }^{6,17}$ A research on Palestinian children found that parental love and proper discipline increase child's resilience by increasing their creativity and cognitive ability. ${ }^{28}$

In respect to the association between family bonding and alcohol drinking; there was an inverse small effect size (statistically insignificant). This inverse relationship was consistent with what is reported in the literatures ${ }^{29}$ it can be explained by that family bonding enhances resilience and the resilient subjects have lower levels of antisocial behaviors (delinquent behavior and substance use), ${ }^{17}$ closeness or a positive relationship with parents reduces the risk of adolescents' substance use, ${ }^{30}$ but a statistical insignificant association indicates that the family bonding might not be sufficient for preventing alcoholism, and that some other factors could be playing a role in alcoholism such as the effect of peer relationships, it is well known that peers, especially friends, play an important role in adolescents' psychosocial development; peer may affect adolescents' behaviors and emotions through socialization processes, a phenomenon more generally referred to as peer influence, ${ }^{31}$ type of media exposure and religious matter of the subjects, in addition to family and community environment which encourage or discourage this habit; a research found that family 
variables have been shown to be related to or predict substance use, as parental use of tobacco or alcohol is a precursor of the onset of smoking or drinking among offspring. ${ }^{30}$

\section{Conclusion and recommendations}

It can be concluded from this study that family bonding during childhood is the most determinant and predictive resilient variable that protects adults against substance use, mental disorders, suicidal attempts, and chronic physical diseases during adulthood. Higher levels of exposure to household dysfunction-abuse have a positive association with substance abuse, suicide attempts, symptoms of mental disorders and chronic physical diseases in adulthood.

High self-esteem score (as an outcome of resilience) has an inverse relationship with scores of depression and anxiety symptoms, suicidal attempts and substance abuse. There is a positive graded relationship between family bonding and self-esteem. Self-esteem for those with primary and intermediate school education is higher than those with university and diploma.

Special national programs are needed to build resilience among society members starting from $\mathrm{KG}$ to universities, including prevention and intervention strategies targeting early adverse childhood experiences and their consequences.

\section{Competing interest}

The authors declare that they have no competing interests.

\section{References}

1. WHO. Adverse Childhood Experiences International Questionnaire (ACE-IQ) 2012. h t t p : / / w w w. W h o. in t / violence injury prevention/violence/activities/ adverse childhood_experiences/en/ (accessed July 2015).

2. Marie-Mitchell A and O'Connor GT. Adverse childhood experiences: translating knowledge into identification of children at risk for poor outcomes. Acad Pediatr 2012;13(1):14-9.

3. Schilling AE, Aseltine HR, Gore S. Adverse childhood experiences and mental health in young adults: a longitudinal survey. $B M C$ Public Health 2007;7:30.

4. Al Diwan JK, Al-Kaseer E, Al-Hadithi T, Al-Hadi A. Mental health of Iraqi adolescents. J Arab Board Med Specialization 2012;11:4955.

5. Duke NN, Pettingell SL, McMorris BJ, Borowsky IW. Adolescent violence perpetration: associations with multiple types of adverse childhood experiences. Pediatrics 2010:125:e778-e86.

6. Clark M. The Family and substance use among
Maltese University Students. $J$ Educ Soc Res 2012;2(3).

7. Orbke S,Smith LH. A developmental framework for enhancing resiliency in adult survivors of childhood abuse. Int $J$ Adv Counselling 2013;35(1):46-56.

8. Fearson JD. Iraq's civil war. Foreign Aff 2007; 86:2-16.

9. Al Shawi AF, Al-Hemiary NF, Al-Diwan JK, Tahir DH. Post-traumatic stress disorder among university students in Baghdad: a preliminary report. Iraq J Comm Med 2011;24: 287-90.

10. Al Hilfi k, Lafta R, Burnham G. Health services in Iraq. Lancet 2013; 381(9870):939-48.

11. WHO. Iraq Mental Health Survey 2006/7 Report. Geneva: World Health Organization, 2009.

12. Al-Jawadi A, Abdul-Rhman S. Prevalence of childhood and early adolescence mental disorder among children attending primary health care centers in Mosal, Iraq: A crosssectional study. BMC 2007;7:274-82.

13. Burnham G, Lafta R, Doocy S, Roberts L. Mortality after 2003 invasion of Iraq: A cross sectional cluster sample survey. Lancet 2006;368 (9545):1421-8.

14. Lafta RK, Aziz ZS, AlObaidi A. Posttraumatic stress disorder among male adolescent in Baghdad. J Psych Abnorm Child 2014;3 (3):121.

15. ICRC Iraq. Giving disabled people a chance to resume a normal life. https://www.icrc.org/eng/ resources/documents/update/2011/iraq-update2011-10-20.htm (accessed July 2015).

16. Sadik S, Abdulrahman S, Bradley M, Jenkins R. Integrating mental health into primary health care in Iraq. Ment Health Fam Med 2011;8:3949.

17. Tiet QQ, Huizinga D, Byrnes FH. Predictors of Resilience Among Inner City Youths. J Child Fam Stud 2010;19:360-78.

18. Lagrange RL, White HR. Age differences in delinquency: A test of theory. Criminology 1985;23:19-45.

19. Kotrlick JW,Williams HA. The Incorporation of effect size in information technology, learning, and performance research. Inf technol Learn Perform J 2003;21(1):1-7.

20. Al Mousawi A. The Prevalence of smoking among Karbala/Iraq University Students in Iraq in 2005. Tob Use Insights 2014;7:9-14.

21. Alhasnawi S, Sadik S, Rasheed M, Baban A, Al -Alak MM, Othman AY, Othman Y, Ismet N, Shawani O, Murthy S, Aljadiry M, Chatterji S,

\footnotetext{
South East Asia Journal of Public Health 2015;5(1):25-32
} 
Al-Gasseer N, Streel E, Naidoo N, Mahomoud Ali M, Gruber MJ, Petukhova M, Sampson NA, Kessler RC; Iraq Mental Health Survey Study Group. The Prevalence and correlates of DSMIV disorders in Iraq Mental health survey (IMHS). World Psychiatry 2009;8:97-109.

22. Felitti VJ, Anda RF, Nordenberg D, Williamson DF, Spitz AM, Edwards V, Koss MP, Marks JS. Relationship of childhood abuse and household dysfunction to many of the leading causes of death in adults: the Adverse Childhood Experiences (ACE) Study. American J Prev Med 1998;14:245-58,

23. Dube SR, Anda RF, Felitti VJ, Chapman DP, Williamson DF, Giles WH. Childhood abuse, household dysfunction, and the risk of attempted suicide throughout the life span, finding from adverse childhood experiences study. JAMA 2001;286(24):3089-96.

24. Herrenkohl TI, Lee JO, Kosterman R, Hawkins JD. Family Influences Related to Adult Substance Use and Mental Health Problems: A Developmental Analysis of Child and Adolescent Predictors. $J$ Adolesc Health 2012;51:129-35.

25. Anda RF, Croft JB, Felitti VJ, et al. Adverse childhood experiences and smoking during adolescence and adulthood. JAMA 1999;282 (17): $1652-58$
26. Engelbrecht A, Jobson L. An investigation of trauma-associated appraisals and posttraumatic stress disorder in British and Asian trauma survivors: the development of the Public and Communal Self Appraisals Measure (PCSAM). Springerplus 2014;3:44.

27. Ahmed AS. Post-traumatic disorders, resilience and vulnerability. Adv Psychiat Treat 2007;13:369-75.

28. Agaibi CE, Wilson JP. Trauma, PTSD and resilience: A review of the literature. Trauma Violence Abuse 2005;6:195-216.

29. Kuendig H, Kutscher E. Family bonding and adolescent alcohol use: moderating effect of living with excessive drinking parents. Alcohol Alcoholism 2006;4(4):464-71.

30. Ledoux S, Miller P, Chooquet M, Plant M. Family structure, parent-child relationships, and alcohol and other drug use among teenagers in France and United Kingdom. Alcohol Alcoholism 2002;37(1):52-60.

31. Giletta M, Scholte RH, Prinstein MJ, Engels RC, Rabaglietti E, Burk WJ. Friendship Context Matters: Examining the domain specificity of alcohol and depression socialization among adolescents. J Abnorm Child Psychol 2012;40:1027-43. 